\title{
Setting standards to promote progress in bio-nano science
}

\begin{abstract}
To the Editor - Standardized minimum information reporting is undeniably valuable. It helps editors, reviewers and readers to access scientific data and experimental details more easily and
\end{abstract} examine them more carefully. It furthermore aids reproducibility, quantitative comparisons, meta-analyses and modelling. In the bio-nano field, a key current question is whether standardized minimum information reporting can help to close the gap between the thousands of bio-nano papers that are published every year and the relatively small number of bio-nano products that have made it to the market.

To evaluate how leaders in the field see the MIRIBEL guidelines (minimum information reporting in bio-nano experimental literature), we contacted around 150 bio-nano experts, and we asked them whether introducing a minimum information reporting standard is valuable (1), necessary (2) and practicable (3); whether it facilitates reproducibility (4), quantitative comparisons (5) and meta-analyses and modelling (6); and whether it promotes scientific integrity (7), scientific progress (8) and clinical progress (9).

As shown in Fig. 1, most of the $n=112$ experts who responded to our request appreciate minimum information reporting. Individual feedback was mixed, though, and ranged from several experts who were fully supportive (all statements maximum score; $n=6$ ) to several who were very critical (all statements minimum score; $n=3$ ). Without being asked to do so, a relatively large number of experts provided several sentences of textual feedback, sharing their general or specific thoughts on minimum information reporting. This feedback was also mixed (see Supplementary Information). Based on the highest percentages of low scores, the most important points of concern turned out to be practicability (3) and contribution to clinical progress (9). The former can be dealt with relatively easily, for example by reducing or refining the reporting requirements. The latter is a harder nut to crack, because creating clinical impact entails much more than standardized data sharing and information reporting.

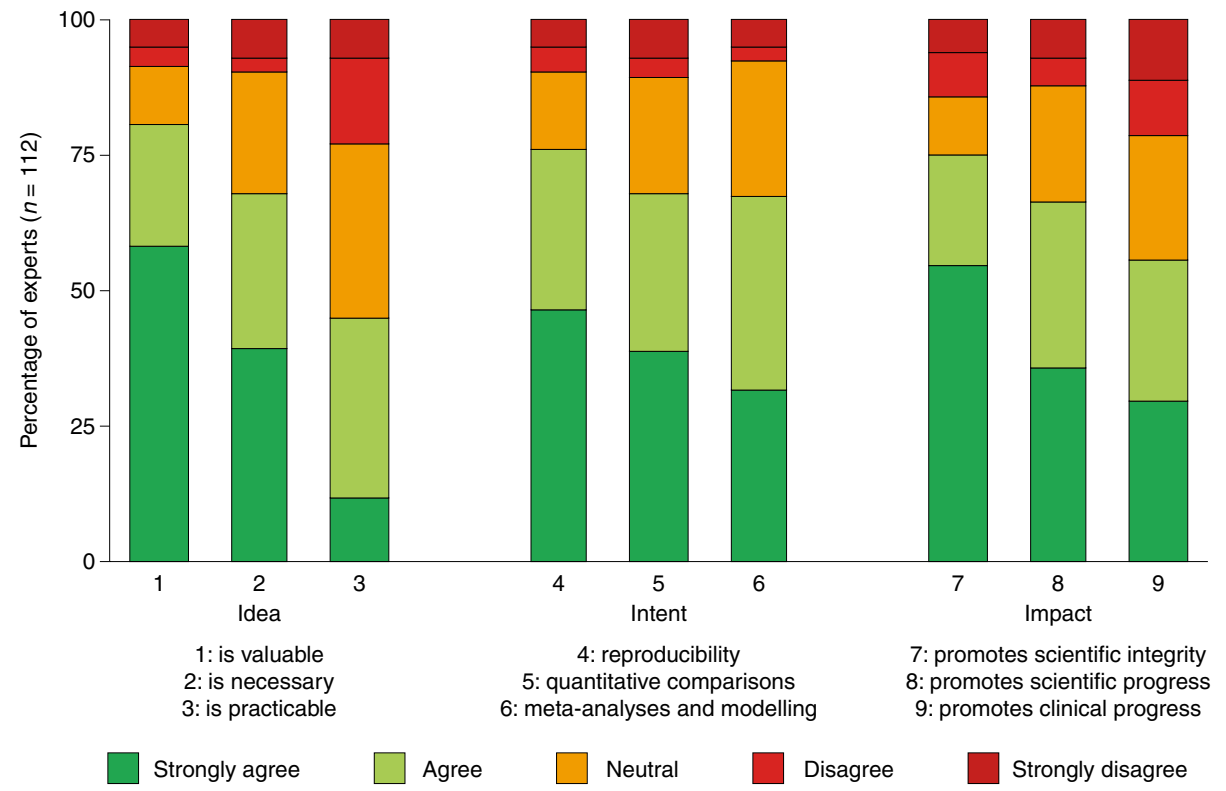

Fig. 1 | Expert assessment. Leading scientists in bio-nanoscience were asked to score the idea, the intent and the impact of introducing a minimum information reporting standard. Specific questions were: whether introducing a standard such as MIRIBEL is valuable (1), necessary (2) and practicable (3); whether it facilitates reproducibility (4), quantitative comparisons (5) and meta-analyses and modelling (6); and whether it promotes scientific integrity (7), scientific progress (8) and clinical progress (9).

We here suggest eMIRIBEL, as an extended version of the proposed minimum information reporting standard, in which, besides information on experimental details, a critical self-assessment is also included, to provide editors, reviewers and readers with to-the-point information on the 'real' added value of the presented bio-nano paper. Does the developed material or method tackle a real medical problem? Does it do so in a rational manner and is the envisaged clinical scenario realistic? How probable is bio-nano product development? What are the limitations of the highly innovative bio-nano platform technology that is being offered for publication?

Certain multidisciplinary journals publish 'significance' sections, and certain medical journals have dedicated 'limitations' and 'research in context' sections. The latter are sometimes subdivided into 'evidence before this study', 'added value of this study' and 'implications of all the available evidence.' In our opinion, it would be wise to consider some of these bigger-picture aspects when intending to set standards to promote progress in bio-nano science - especially if the (cl)aim is medical application.

Twan Lammers ${ }^{1,2,3}$ and Gert Storm ${ }^{2,3}$ ${ }^{1}$ Department of Nanomedicine and Theranostics, Institute for Experimental Molecular Imaging, RWTH Aachen University Clinic, Aachen, Germany. ${ }^{2}$ Department of Pharmaceutics, Utrecht University, Utrecht, the Netherlands. ${ }^{3}$ Department of Targeted Therapeutics, University of Twente, Enschede, the Netherlands.

e-mail:tlammers@ukaachen.de; g.storm@uu.nl

Published online: 3 July 2019

https://doi.org/10.1038/s41565-019-0497-8

Additional information

Supplementary information is available for this paper at https://doi.org/10.1038/s41565-019-0497-8. 